\title{
Management of Temporomandibudar Joint Ankylosis with Costo-Chondral Graft Application: Case Report and Review of Literature

\author{
Nikolaos Kolomvos ${ }^{1 *}$, Agamemnon Chliaoutakis ${ }^{2}$ and Nikolaos Papadogeorgakis ${ }^{3}$
}

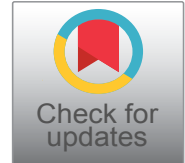

${ }^{1}$ Assistant Professor, Department of Oral and Maxillofacial Surgery, EVANGELISMOS General Hospital, Dental School, University of Athens, Greece

${ }^{2}$ Postgraduate Student, Department of Oral Surgery, EVANGELISMOS General Hospital, Dental School, University of Athens, Greece

${ }^{3}$ Professor Emeritus, Department of Oral and Maxillofacial Surgery, EVANGELISMOS General Hospital, Dental School, University of Athens, Greece

*Corresponding author: Nikolaos Kolomvos, Assistant Professor, Department of Oral and Maxillofacial Surgery, EVANGELISMOS General Hospital, Dental School, University of Athens, 2 Thivon Str., Goudi, Athens, 115 27, Athens, Greece, Tel: +30-210-7461288

\section{Introduction}

The term ankylosis of temporomandibular joint (TMJ) is derived from the Greek word meaning stiffening of a joint due to disease process and referred to describe a permanent movement disfunction of the mandible caused by the fusion of joint surfaces by bone, fibrous or mixed tissue, bilateral or unilateral [1-3]. When the cause of the ankylosis is extra-articular is referred to as pseudoankylosis or false ankylosis, while when caused by intra-articular problems referred to as true ankylosis [2]. Extra-articular ankylosis may be due to the synostosis of the jaws after inadequate surgical treatment of the condylary area, development of fibrous tissue in the muscle masses which surrounding jaw (after organization of hematomas, burn or radiotherapy, submucosal fibrosis and ossified myositis), as well as other causes that create mechanical barriers to the movement of the mandible (e.g. trauma, fractures of the zygomatic arch when the descending segments are inserted in the course of the coronoid process, by hyperplasia of the coronoid process) and due to region's sizable neoplasms $[2,3]$. The causes that lead to a true TMJ ankylosis may be congenital (e.g. due to obstetric in- jury) or acquired $[4,5]$. Various acquired pathological conditions of the TMJ may lead to perforation and/or degeneration of the articular disc and lead eventually into TMJ ankylosis. Acquired etiologic factors include lesions, trauma and mainly intra-articular fractures of the condyle, local and systemic inflammatory conditions, neoplasms, TMJ infection and arthritis that lead to the synostosis of the TMJ (infectious or chronic inflammatory arthritis and mainly ankylotic spondylo-arthritis, rheumatoid arthritis, fibrosis and coronoid process hyperplasia). The maximum opening of the mouth is 3-4 mm when true ankylosis occurs, while in extra-articular ankylosis is $>4 \mathrm{~mm}$ and $<30$ $\mathrm{mm}[2,3,6]$.

Therapeutic treatment of TMJ ankylosis requires excision and removal of the ankylotic mass and involved bone, as well as reconstruction of the TMJ with different types of materials (autografts or allografts) [7]. Reconstruction of the TMJ with autogenous element represents one of the most intriguing and challenging operations in all of the bone grafting $[6,8]$.

Purpose of this case report is to present the surgical management of a unilateral TMJ ankylosis and recent literature review. Surgical treatment included 
resection of the newly formed bone mass, replacement of the condyle with the use of costo-chondral graft and replacement of the articular disc with the temporal fascia.

\section{Case Report}

A thirty-seven-year-old male patient presented to the Oral and Maxillofacial Clinic of "Evangelismos" Hospital complaining of the inability to open his mouth since his birth. When the medical history was received, it was found that the unilateral TMJ ankylosis was of congenital etiology due to the operative instrumental delivery. The patient had undergone corrective surgery before years, but the ankylosis had relapsed. During the clinical examination the maximum opening of the mouth was 2-3 mm, while the lateral movements of the mandible were depressed. The development of the anterior region of the maxilla was disturbed and formed in a V-shaped dental arch due to the use of a feeding instrument from neonatal age (Figure 1). A conventional radiographic examination was performed including a panoramic X-ray, as well as a CT and three-dimensional CT scan. Radiological examinations showed that the ankylosis was in the right TMJ due to the development of bone between the condyle and the articular fossa including anterior and posterior articular tubercle (Figure 2).

Surgical management of the TMJ ankylosis was decided in order to achieve rehabilitation of the mandible

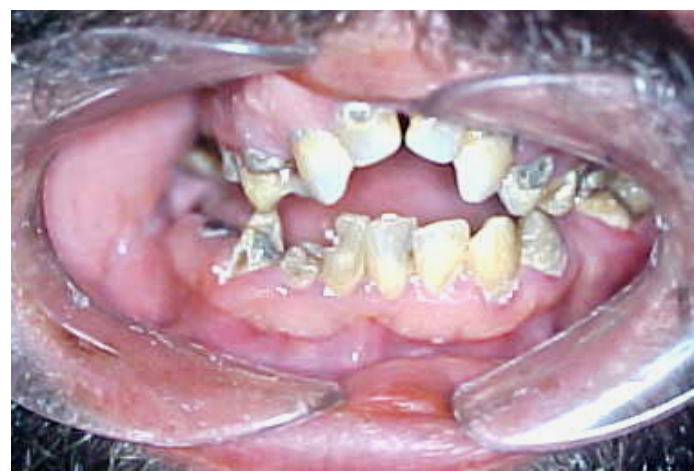

Figure 1: Limited opening of the mouth and the V-shaped formation of the maxilla. and mastication. A preauricular incision with temporal expansion performed and revealed the articular fossa and the TMJ (Figure 3). Resection of the newly formed bone mass and resection of the coronoid process was performed and the ankylosis of the TMJ was dissolved. The reconstruction of the condyle was achieved with a costo-chondral graft from the $6^{\text {th }}$ rib of the patient (Figure 4), which was fixated in the ramus (Figure 5). The adjustment of the articular fossa performed next, followed by the mobilization of the temporal fascia. The temporal fascia sutured in the region replacing the articular disc and preventing new ankylosis. The function of the joint was tested and the maximum mouth opening showed nearly $40 \mathrm{~mm}$ (Figure 6).

Postoperatively, analgesics and non-steroidal anti-in-

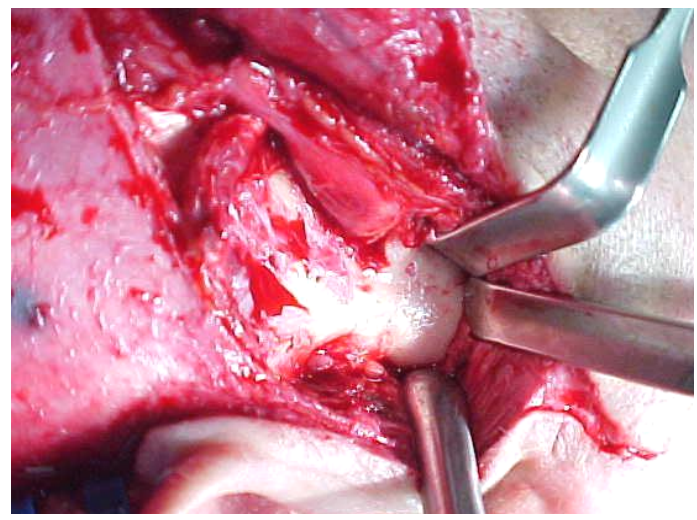

Figure 3: Incision showing the TMJ and the newly formatted bone causing the ankylosis.

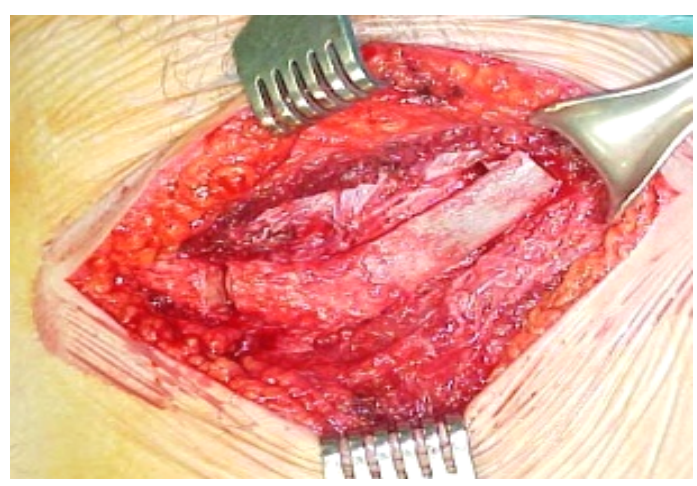

Figure 4: Costo-chondral graft from the $6^{\text {th }}$ rib.

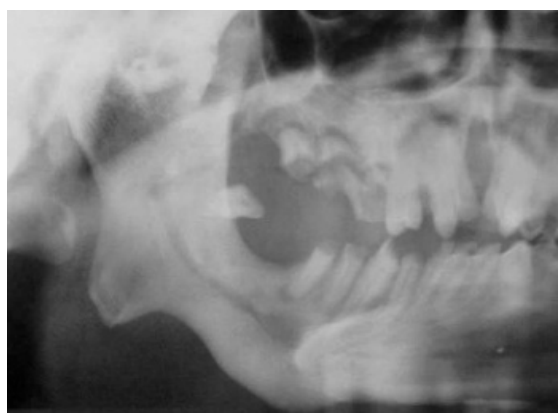

A

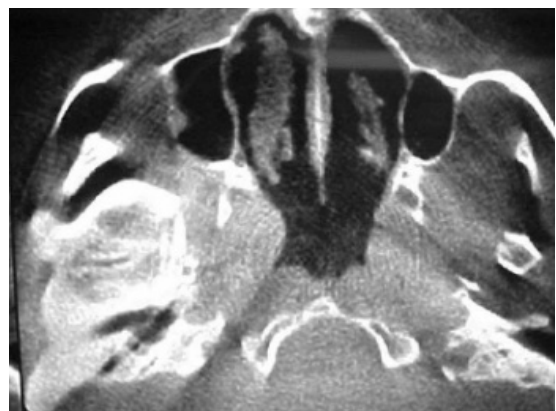

B

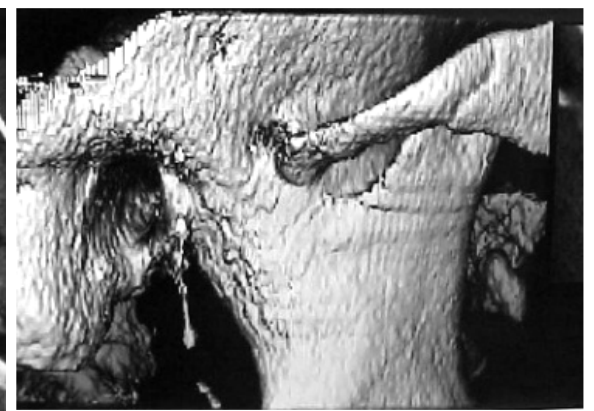

C

Figure 2: Radiographic examination of the patient. A) Panoramic X-ray; B) CT-scan; C) 3D-reconstruction image. 


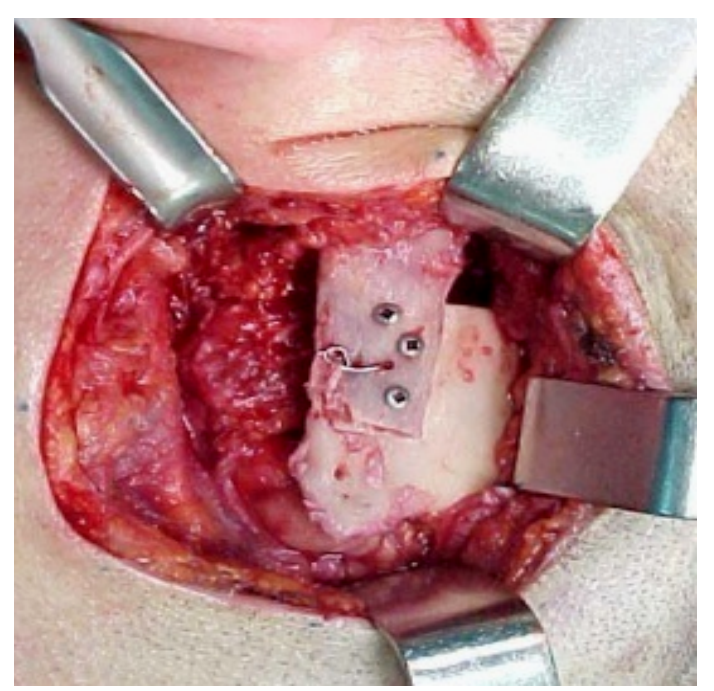

Figure 5: Reconstruction of the condyle with costo-chondral graft.

flammatory were administered and application of hot patches, while the patient followed an active physiotherapy program including active and passive mouth-opening exercises, anterior-posterior and lateral movements of the mouth, which began on the second postoperative day and continued for 6 months. Ten years after the surgical treatment and rehabilitation of the dental arches, the patient showed no recurrence and the postoperative result was maintained stable.

\section{Discussion}

Temporomandibular joint (TMJ) ankylosis is a degenerative disease accompanied by pain and dysfunction of the mandible and is a condition that may cause chewing, digestion, speech, esthetic, hygienic and psychological disorders $[2,8]$. In children, TMJ ankylosis usually presents with facial asymmetry, disturbance of the growth of the facial skull and volatile form when bilaterally is detected $[4,9]$.

According to the tissue growing in the intracapsular structures of the joint, ankylosis is classified as fibrous, bony or mixed and divided into 4 types: Three of them concern total ankylosis, while the $4^{\text {th }}$ refers to a partial ankylosis (lateral). They are distinguished by the following: $[8,10]$.

- Type I: The head of the condyloid process is visible, but significantly deformed with the fibro adhesions making TMJ movement impossible.

- Type II: The deformed head of the condyloid process and the articular surface are in contact in the anterior and posterior region of the TMJ, while the medial part of the surface of the condylar head remains undamaged.

- Type III: The ankylotic mass involves the mandibular ramus and zygomatic arch and an atrophic and displaced fragment of the anterior part of the condylar head is in a medial location.

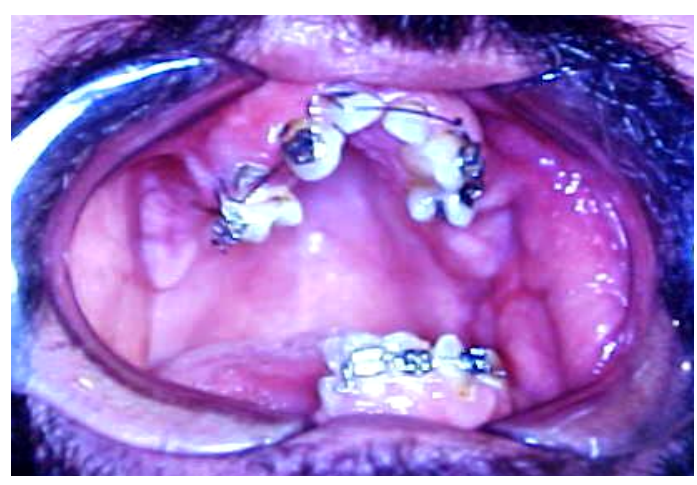

Figure 6: Mouth opening immediate after surgery.

- Type IV: TMJ is completely obliterated by bony ankylotic mass growing between the mandibular ramus and cranial base.

Conventional radiographs (mainly panoramic and Town's radiography) are very helpful in the diagnosis other than the history (mainly for the exclusion of malignancy) and the clinical examination, although clear information on the extent of the ankylosis are taken mainly with CT scan and three-dimensional CT scan [2] Main criterion for the diagnosis of ankylosis is the distance between the central incisors of the upper and lower jaw, which can range from 0-30 mm. In unilateral ankylosis there is the possibility of some degree opening of the mouth, while in bilateral ankylosis the movement of the mouth is less or completely impossible [8]. Differential diagnosis should be made by other pathological conditions that cause a restriction of the mobility of the mandible (trismus). Specifically, limited mobility of lower jaw may be due to an infection of the surrounding tissue (due to infection of an anatomical space, pericoronitis etc.), lesions, swelling of the muscles, after local anesthesia of the Inferior alveolar nerve (formation and organization hematoma), syndrome of masticator myalgia-dysfunction, tetanus or hysteric trismus [2].

Treatment requires a combination of surgery and physiotherapy in order to restore the functionality of the mandible and prevent relapse $[1,2,6]$. A number of treatments and many techniques have been described in the literature for the restoration of both the condyle and the articular fossa, including simple arthroplasty, interposition arthroplasty using temporal muscle fascia or ear cartilage and reconstruction of the joint with autogenous tissue harvested from the patient $[8,9,11]$. Joints reconstructed with alloplastic materials have been subject to complications such as acute infection and chronic inflammatory problems as a result of foreign-body reaction with the immune system [12]. The most commonly used technique is arthroplasty with transportation of the temporal fascia and/or muscle. A management protocol for temporomandibular joint (TMJ) ankylosis consisting: $[1,8,9]$. 
- Application of general nasotracheal anesthesia or tracheostomy if there is limited mouth access.

- Extensive resection of the condyle and the bone mass responsible for the ankylosis according to its volume. Condylectomy gives good results in selected cases, where the duration of ankylosis is minimal. Bone resection is limited (maximum $1 \mathrm{~cm}$ ) to avoid excessive decrease in the height of the ramus and in order to avoid recurrences, while there are other dangers due to the proximity of the area with the base of the skull, the personal nerve and the carotid branch [13].

- Unilateral or bilateral coronoidectomy.

- Replacement of condyloid process with chondro-osseous graft (in children with iliac bone graft [9]). Modification of this technique is the use of resected bone instead of chondro-osseous graft $[11,14]$. Costo-chondral graft decreases the incidence of reankylosis after gap arthroplasty and keeps the proper ramus height, while the advantages are biological compatibility and functional adaptability [15]. Potential problems with the use of costo-chondral graft include fracture, donor site morbidity and the variable growth behavior of the graft [11]. Clinically, the graft prevents jaw deviation or production of an open bite and in children is an active growth centre $[4,16]$.

- Smoothening the newly formed articular fossa.

- Replacement of the articular disc with a flap of the temporal fascia and/or muscle and/or cartilage [17].

- Rigid fixation and good connection between the tissues.

- Early mobilization and aggressive physiotherapy.

The main problem for the restoration of the morphology and function of the TMJ is the replacement of the articular disc, because its removal without its replacement can lead to reduced mobility of the condyle and limited mouth opening, disturbances of the occlusion, degenerative lesions of the joint and long-term bone development between the articular surfaces [14,18]. In advanced cases it is necessary to remove the articular disc and replace the disc in order to prevent the degeneration of the elements of the TMJ $[6,14]$. A relapse rate of approximately $50 \%$ in patients who did not replace the disc after the broad resection of the responsible tissues and $0 \%$ in patients with replaced articular disc. The criteria for selecting the reconstruction material of the condyle and the articular disc are the aesthetic result in cases of graft taken from another area, the risk of infection of the donor site and the position, the biocompatibility and durability of the material, as well as the risk of recurrence [8].

Several materials and grafts have been used to re- store the articular disc of TMJ after the dissolve of the ankylosis. First autologous grafts such as temporal fascia alone or in combination with the muscle and periosteum, lyophilized meningeal graft, skin graft, the auricular cartilage graft and substitute grafts such as collagen of bovine origin $[5,8,19,20]$. The technique including the use of temporal fascia and muscle reported higher success rates. The lower part of the temporal muscle and its fascia offer an excellent graft for replacing the disc, preventing the development of degenerative lesions and maintaining dental occlusion. It is used as an intermediate layer between the articular surfaces and also fulfills the empty space interleaved between articular fossa and condyle [18]. Specifically, after resection of the bone mass responsible for the ankylosis, the temporal fascia and muscle are used to fill the space of the articular fossa preventing the revascularization between the bony surface of the ramus and the articular fossa [8]. The flap is malleable and retains its vitality after its transportation into the new position. Its advantages are lack of toxicity and antigenic properties, easily moved to the receiver position, shows good stability, while the most important advantage is the reduction of surgical time due to the proximity of the donor to the recipient site. Also, the temporal fascia in contact with the articular fossa reduces the friction between the articular surfaces thus maintaining the functionality of the joint, it offers better mobility in the condyle and the function of the flap resembles that of the articular disc. In patients managed with this technique it is observed functional and occlusal stability, the healing is promoted, pain decreases and helps the resolution of the chronic inflammatory elements from the area. However, a small degree of degeneration and atrophy of the muscle can occur, which can lead to new ankylosis $[17,18]$. The skin as a graft offers many satisfactory results. It has the advantage that it is autologous, so there is no risk of a foreign body reaction, it is resistant to pressure and distention and can be directly postoperatively to be "charged", prevents the synostosis of the articular surfaces and the re-ankylosis as it is transformed into scar tissue that is hypokinetic. The disadvantage is that a remote donor position is required, it has an incompatible texture and is placed as a free graft on a relatively rude bed that is subject to movement and contamination can occur. A total thickness graft with dimensions of approximately $50 \times 35 \mathrm{~mm}$ can be taken from the posterior surface of the fin of the ear and sutures, so that the epidermis is toward temporal fossa $[8,19]$.

Alloplastic materials have also been used as a permanent or temporary solution. Alloplastic joints make it possible to reproduce more closely the natural joint anatomy, restoring the vertical height, decreasing surgery time, and reducing the rate of recurrences [21]. The criteria for failure to replace the disc of the TMJ are pain during a joint operation, reduced mobil- 
ity of the mandible, disturbances of the occlusion and radiographic findings due to bone lesions (erosions and displaced bone mass formation) $[7,12,20]$. The TMJ may also be filled with granular tissue, corrosion of the zygomatic arc may occur, perforation of the tip of the articular fossa, absorption of the condyloid process due to extensive corrosion, fibro-osseous ankylosis and complete displacement of the material or fragmentation [21]. Even metallic implants materials have been used in cases of ankylosis. The use of alloplastic implant materials was first applied by Gordon in 1955 (used an only graft on the condyle made of polyethylene), while before discectomy was performed without replacing the disc. These materials were used in order to prevent the reduction of the height of the ramus, the reduced mobility of the mandible, the disturbances of occlusion and the degeneration of the elements of the joint [20]. Complications that may occur with the use of metallic implants are the foreign body reaction, bone erosion accompanied by pain, decreased mobility of the mandible, disturbances of occlusion, lymphadenopathy and reduced stability of the implant and fracture of the implant [18].

Regular recall of the patient is required, while relapse may occur mainly in cases of postoperative bleeding that promotes osteogenesis in the area, and which is avoided by fast kinesitherapy $[3,19]$. The major problem of surgical correction of the TMJ ankylosis is the high recurrence rate and the failure to maintain the result, therefore immediate postoperative kinesiotherapy is required from the $2^{\text {nd }} d a y$, which includes passive and energetic exercises of the mouth opening and occlusion, anterior-posterior and lateral movements of the mouth and lasts several months until no further improvement is expected [2,9].

\section{Conclusions}

The development of TMJ ankylosis may be congenital or acquired etiology $[4,5]$. In order to manage an ankyloses a detailed history is required (mainly to block malignancy) and a comprehensive clinical and radiographic examination. Surgical resection of the bone (approx. $2 \mathrm{~cm}$ ) is performed to prevent relapse, and resection of the coronoid process on the entire side is necessary, while on the opposite side only if necessary. The functional restoration is achieved if a graft that mimics the articular disc is placed [1]. In conclusion, it is of great importance the immediate postoperative kinesitherapy and regular patient recall program [3].

\section{References}

1. Kaban LB, Perrott DH, Fisher K (1990) A protocol for management of temporomandibular joint ankylosis. J Oral Maxillofac Surg 48: 1145-1151.

2. Güven $O$ (2000) A clinical study on temporomandibular joint ankylosis. Auris Nasus Larynx 27: 27-33.
3. Manganello-Souza LC, Mariani PB (2003) Temporomandibudar joint ankylosis: Report of 14 cases. Int J Oral Maxillofac Surg 32: 24-29.

4. Ohno K, Michi K, Ueno T (1981) Mandibular growth following ankylosis operation in childhood. Int J Oral Surg 10: 324-328.

5. von Domarus H, Scheunemann H (1990) Congenital prearticular temporo-mandibular ankylosis in two siblings. J Cranio-Maxillofacial Surg 18: 299-303.

6. Loveless TP, Bjornland T, Dodson TB, Keith DA (2010) Efficacy of Temporomandibular Joint Ankylosis Surgical Treatment. J Oral Maxillofac Surg 68: 1276-1282.

7. Saeed NR, Hensher R, McLeod NMH, Kent JN (2002) Reconstruction of the temporomandibular joint autogenous compared with alloplastic. Br J Oral Maxillofac Surg 40: 296-298.

8. Chossegros C, Guyot L, Cheynet F, Blanc JL, Cannoni P (1999) Full-thickness skin graft interposition after temporomandibular joint ankylosis surgery. Int $\mathrm{J}$ Oral Maxillofac Surg 28: 330-334.

9. Matukas VJ, Szymela VF, Schmidt JF (1980) Surgical treatment of bony ankylosis in a child using a composite cartilage-bone iliac crest graft. J Oral Surg 38: 903-905.

10. Zhi K, Ren W, Zhou H, Gao L, Zhao L, et al. (2009) Management of temporomandibular joint ankylosis: 11 years' clinical experience. Oral Surgery, Oral Med Oral Pathol Oral Radiol Endodontology 108: 687-692.

11. Demir Z, Velidedeoglu H, Sahin U, Kurtay A, Coskunfirat OK (2001) Preserved costal cartilage homograft application for the treatment of temporomandibular joint ankylosis. Plast Reconstr Surg 108: 44-51.

12. Mercuri LG, Swift JQ (2009) Considerations for the Use of Alloplastic Temporomandibular joint Replacement in the Growing Patient. J Oral Maxillofac Surg 67: 1979-1990.

13. Weinberg S, Kryshtalskyj B (1992) Facial nerve function following temporomandibular joint surgery using the preauricular approach. J Oral Maxillofac Surg 50: 1048-1051.

14. Medra AM (2005) Follow up of mandibular costochondral grafts after release of ankylosis of the temporomandibular joints. Br J Oral Maxillofac Surg 43: 118-122.

15. Macintosh RB (2000) The use of autogenous tissues for temporomandibular joint reconstruction. J Oral Maxillofac Surg 58: 63-69.

16. Himanshu Sharma, Shouvik Chowdhury, Anuradha Navaneetham, Sonal Upadhvav, Sarwar Alam (2015) Costochondral Graft as Interpositional material for TMJ Ankylosis in Children: A Clinical Study. J Maxillofac Oral Surg 14: 565-572.

17. Su-Gwan K (2001) Treatment of temporomandibular joint ankylosis with temporalis muscle and fascia flap. Int $\mathrm{J}$ Oral Maxillofac Surg 30: 189-193.

18. Umeda H, Kaban LB, Anthony Pogrel M, Stern M (1993) Long-term viability of the temporalis muscle/fascia flap used for temporomandibular joint reconstruction. J Oral Maxillofac Surg 51: 530-533.

19. Dimitroulis G (2004) The interpositional dermis-fat graft in the management of temporomandibular joint ankylosis. Int J Oral Maxillofac Surg 33: 755-760.

20. Mercuri LG, Ali FA, Woolson R (2008) Outcomes of Total Alloplastic Replacement With Periarticular Autogenous Fat Grafting for Management of Reankylosis of the Temporomandibular Joint. J Oral Maxillofac Surg 66: 1794-1803.

21. Matsuura H, Miyamoto H, Ogi N, Kurita K, Goss AN (2001) The effect of gap arthroplasty on temporomandibular joint ankylosis: An experimental study. Int J Oral Maxillofac Surg 30: 431-437. 